\title{
Broad applications of single-cell nucleic acid analysis in biomedical research
}

\author{
Michael Wigler*
}

Recent publications have demonstrated the feasibility and utility of sequencing DNA or RNA from single cells $[1,2]$. The power of these tools lies in their ability to reveal in mixtures of cells the identity and state of subpopulations. The advantage of single-cell analysis over merely deep sequencing, whether for DNA or RNA, lies in the ability of the former to isolate differences and to phase possibly multiple differences within subpopulations. The future applications of these technologies are the topic of this brief article.

I will assume throughout that in the near future assessing both DNA and RNA content simultaneously from hundreds to thousands of single cells will be quantitatively accurate, as complete as needed, and affordable. It follows from these assumptions that knowledge of chromatin configuration and DNA methylation in single cells will also be attainable. I will not consider what is possible by assaying the protein content of single cells, either by microscopy or mass spectroscopy, or by using methods not yet invented. Also, I will not focus on the many applications of this technology in oncology, such as in outcome research and early detection, as these applications are ongoing and have been widely discussed elsewhere [3]. Instead, I want to speculate on basic applications to cell biology, theory of disease and diagnosis.

\section{Basic cell and organismal biology}

Much of what we know about cell biology is derived from perturbation experiments. An agent - physical, chemical or genetic - acts upon a cell and elicits a sequence of reactions, whether metabolic, molecular or structural. Unless this response is monitored by microscopy or some other method for observing single cells, the response is measured in a population of cells. Even superficially homogeneous populations contain cells in different phases of the cell cycle, or that have stochastic variance, and thus may differ in response to a perturbing agent. The temporal chain of reactions is often muddied.

*Correspondence: wigler@cshl.edu

Cold Spring Harbor Laboratory, PO Box 100, Cold Spring Harbor, NY, 11724, USA
High-throughput single-cell nucleic acid analysis closes this gap, allowing the analysis of many single cells in a population, each responding to the perturbation. Each cell is a snapshot in the temporal chain of events, so mathematical and statistical methods are needed to piece together the response pathways [4]. Such analysis can reveal the causal linkage between events that were merely correlated before. Examples of potential applications abound, including studying the cellular response to DNA damage, hormones, contact with another cell, release from nutrient limitation and so forth.

In addition, the application of single cell genomic analysis should enable a clearer assessment of the nature of regulatory control. How tightly regulated are RNA levels from cell to cell? Is RNA expression (amount and splicing) monoallelic [5]? Because - under the assumptions outlined above - chromatin configuration and DNA methylation from single cells will also be attainable, we can anticipate being able to distinguish cause and effect when both transcription and chromatin change.

Any organ is made up of subpopulations of cell types, such as the various components of the blood, endothelial cells, stem cells and their descendants with varying degrees of functional specialization. There is not a complete set of markers identifying all the population subtypes. Clustering single-cell RNA profiles is likely to yield a deeper recognition of cell types and to arm us with new tools for recognizing these cells. Most important among these are markers for stem cells and armed with such markers, single-cell analysis does allow the inspection of rare subpopulations [2].

For a cell of known type, we can also speak of its 'state'. Cells of the same type may vary in state as they react to soluble factors, neighboring cells or synaptic inputs, transit through the cell cycle, and so on. In many of these cases, the state of the cell may be recognized from the expression profile, and moreover the state may help us recognize which influences are acting on the cell. Thus, single-cell methods can be applied to such diverse problems as detecting the state of the stem cells in a given organ or understanding the transcriptional reaction of neurons to stimuli. 


\section{Pathobiology and diagnosis}

Once armed with expectations about the normal state of tissues, we can use these expectations to investigate various disorders, such as diabetes and cancer. For example, in the latter, one can assess the cell types of host stroma, and their potential functions at the tumor site. Less obvious is the opportunity to analyze the basis of the medical disorders that result from the dysfunction of stem cells. Although speculative, it is likely that acquired problems of stem cells lie at the heart of many disorders of aging. These acquired problems can result from loss of DNA methylation or other epigenetic markers, and/or from accumulated somatic mutation such as loss of heterozygosity that can unmask existing genetic vulnerabilities. Acquired autoimmunity, psoriasis or degenerative conditions, such as amyotrophic lateral sclerosis may be examples. Using single-cell methods, and our ability to distinguish cell types by their expression patterns, it should be possible to directly search for the presence of pathogenic somatic mutations in subtypes of dysfunctional tissues.

In the future many tissue assays could be performed by single-cell methods, minimizing the necessary sample size and thereby lessening the stress of the biopsy procedure on the patient. The opportunities for cancer diagnosis from biopsies or fluid samples are clear. The most extreme example is pre-implantation genetic screening, where an assay of a single cell might guide embryo selection. A less obvious but broader set of diagnostics could be based on analysis of blood cells. The blood is the highway of the body, and it is likely that the cells that traffic there reflect specific defense and repair functions, as well as hints of proliferative disorders. For example, the state of immune cells might reflect the type of invading pathogen that the body is fighting, and damage to a particular organ might be reflected in the type of stem cell that is being recruited from the marrow. Learning to interpret the state and identity of cells in the blood from the RNAs they produce should be seen as a logical next step in the progression of blood-based clinical testing.

Clearly, there is far more to a cell than its DNA and RNA content. Nevertheless, high-throughput single-cell nucleic analysis has many applications to basic biomedical research. In time, these methods will alter medical care by maximizing the utility of minimal biopsy samples, and thereby improving treatment with earlier diagnosis using less invasive procedures.

\section{Competing interests}

The author declares that he has no competing interests.

\section{Acknowledgements}

I wish to acknowledge useful discussions with many colleagues, including Nick Navin, Jim Hicks, Jude Kendall, Mike Ronemus, Zhu Zhu, Dan Levy, Larry Norton and Josh Huang. I am grateful for generous support from the Department of Defense (grant number W81XWH-11-1-0747) and the Simons Foundation.

Published: 30 October 2012

\section{References}

1. Navin N, Kendall J, Troge J, Andrews P, Rodgers L, Mclndoo J, Cook K, Stepansky A, Levy D, Esposito D, Muthuswamy L, Krasnitz A, McCombie WR, Hicks J, Wigler M: Tumour evolution inferred by single-cell sequencing. Nature 2011, 472:90-94.

2. Glotzbach JP, Januszyk M, Vial IN, Wong VW, Gelbard A, Kalisky T, Thangarajah H, Longaker MT, Quake SR, Chu G, Gurtner GC: An information theoretic, microfluidic-based single cell analysis permits identification of subpopulations among putatively homogeneous stem cells. PLoS One 2011, 6:e21211.

3. Navin N, Hicks J: Future medical applications of single-cell sequencing in cancer. Genome Med 2011, 3:31.

4. Ramakrishnan N, Tadepalli S, Watson LT, Helm RF, Antoniotti M, Mishra B: Reverse engineering dynamic temporal models of biological processes and their relationships. Proc Nat/ Acad Sci U S A 2010, 107:12511-12516.

5. Chess $A$ : Mechanisms and consequences of widespread random monoallelic expression. Nat Rev Genet 2012, 13:421-428.

doi:10.1186/gm380

Cite this article as: Wigler M: Broad applications of single-cell nucleic acid analysis in biomedical research. Genome Medicine 2012, 4:79. 